\title{
Persistence with Basal-Bolus Insulin Therapy in Patients with Type 2 Diabetes Mellitus and Effect on Clinical and Economic Outcomes: A Retrospective Claims Database Study
}

\author{
Steven V. Edelman, MD; Anastasia Ermakova, PharmD; Yan Xiong, MS; \\ Ray Sieradzan, PharmD; and Stephanie D. Taylor, PhD
}

\begin{abstract}
BACKGROUND: Persistence with multiple daily insulin injections (MDI) may be challenging for patients with type 2 diabetes (T2DM). However, limited information is available regarding the effect of persistence with MDI on outcomes.

OBJECTIVE: To evaluate persistence with basal and bolus insulin therapy and assess its relationship with clinical and economic outcomes in a realworld setting.
\end{abstract}

METHODS: This retrospective matched cohort study used 2012-2015 data from multiple U.S. commercial health plans (IBM MarketScan). Patients with T2DM aged 18-64 years with $\geq 2$ basal and $\geq 2$ bolus insulin claims during a 12-month period were eligible for inclusion if they had 18 months of continuous health plan enrollment (6-month baseline and 12-month post-index). Persistence during 12 months post-index was defined using 2 methods: (a) method 1, $\leq 90$-day gaps in both basal and bolus insulin claims and (b) method 2, $\geq 1$ basal and $\geq 1$ bolus insulin claim every quarter (every 90 days) for 4 consecutive quarters after index bolus claim. Propensity score matching was used to match persistent and nonpersistent method 2 cohorts. Mean per-patient all-cause and diabetes-related medical costs (2015 U.S. dollars, excluding outpatient drugs) and health care resource use (HCRU) were calculated. For patients with hemoglobin A1c (A1c) values during baseline and post-index months 10-12, treatment success was defined as (a) A1c decrease from baseline of $\geq 1 \%$ and/or (b) baseline $A 1 c \geq 7 \%$ with post-index $A 1 c<7 \%$. Baseline characteristics of matched cohorts were compared using standardized mean differences (SMDs). Outcome variables were compared using t-tests, chi-square tests, and generalized linear models.

RESULTS: Characteristics of 12,882 eligible patients and 12-month persistence rates were similar as defined by method $1(22.4 \%)$ and method 2 (21.1\%). After matching, the method 2 cohorts included 2,723 and 8,169 persistent and nonpersistent patients, respectively, with well-balanced baseline characteristics (mean age 53 years; $58 \%$ men; all SMDs $<0.1$ ). All-cause annual medical costs were lower for the persistent cohort (mean $\$ 13,499$ vs. $\$ 17,362 ; P<0.0001)$, as were annual diabetes-related costs (mean $\$ 6,392$ vs. $\$ 8,376 ; P<0.0001$ ). In persistent versus nonpersistent cohorts, $11 \%$ versus $15 \%$ of patients, respectively, experienced $\geq 1$ hospitalization; $21 \%$ versus $24 \%$, respectively, had $\geq 1$ ED visit; $9 \%$ versus $12 \%$, respectively, experienced $\geq 1$ diabetes-related hospitalization; and $13 \%$ versus $15 \%$, respectively, had $\geq 1$ diabetes-related ED visit $(P \leq 0.005$ for all). Mean baseline A1c was similar in persistent and nonpersistent cohorts (9.7\% vs. $9.6 \%$, respectively; $P=0.63)$. Persistence with MDI was associated with greater mean reduction in A1c $(-1.3 \%$ vs. $-0.8 \%$, respectively; $P=0.006)$ and greater percentages of patients achieving treatment success ( $55 \%$ vs. $39 \%$, respectively, for nonpersistent; $P=0.009$ ).

CONCLUSIONS: Poor persistence with basal-bolus insulin therapy over 12 months of follow-up was prevalent and was associated with greater medical costs, greater HCRU, and poorer glycemic control than for patients who were persistent. Interventions are needed to improve persistence with insulin therapy and aid patients with T2DM to achieve glycemic control.

J Manag Care Spec Pharm. 2019;25(12):1420-31

Copyright $\odot 2019$, Academy of Managed Care Pharmacy. All rights reserved.

\section{What is already known about this subject}

Persistence with basal and bolus insulin therapy is challenging for patients with type 2 diabetes (T2DM) and requires further investigation to assess its effect on clinical and economic outcomes

Real-world data currently available suggest that persistence with basal insulin for T2DM is associated with improved clinical outcomes and lower health care resource use (HCRU), while nonpersistence with basal insulin therapy is associated with increased health care costs.

Data are limited regarding persistence with multiple daily insulin injection (MDI) regimens for patients with T2DM who require both basal and bolus insulin therapy to maintain glycemic control.

\section{What this study adds}

This study used 2 different methods to define persistence over 12 months for both basal and bolus insulin (MDI) therapy in a large real-world patient population with T2DM: (a) prescription claim gaps of $\leq 90$ days and (b) prescription claims every 90 days for at least 4 quarters

A novel aspect of the study is the concurrent determination of persistence for both basal and bolus insulin.

Persistence with MDI was low according to both definitions (21\%-22\%), and the association of poor persistence with worse outcomes, including greater mean annual medical costs, greater HCRU, and poorer glycemic control, highlights the need for interventions to improve insulin persistence in patients with T2DM.

$\mathrm{P}$ romoting adherence to and persistence with long-term therapies is an important goal to address the burden of chronic conditions for patients, their families, and health care systems. ${ }^{1-3}$ Diabetes is one of the most common chronic conditions in the United States, where 1 of every 4 health care 
dollars is spent on the care of patients with diagnosed diabetes, and the prevalence and medical costs of diabetes are projected to continue rising. ${ }^{4.5}$ Of the estimated 30.3 million people (>9\% of the U.S. population) with diabetes, 90\%-95\% have type 2 diabetes mellitus (T2DM), ${ }^{6}$ a chronic, progressive condition characterized by hyperglycemia and requiring regular adjustments to therapy to maintain glycemic control.

Glycemic control is an integral component of the multifaceted management of T2DM, aimed at preventing diabetesrelated complications, such as cardiovascular and microvascular disease. ${ }^{7}$ In addition, much of the costs of T2DM are attributable to these complications. ${ }^{8}$ The recommended intensification of T2DM pharmacotherapy required to maintain glycemic control over time often requires the progression from a single oral antidiabetic drug (OAD) to dual or triple combination OAD therapy and/or to the addition of injectable therapies, beginning with long-acting basal insulin and/or a glucagon-like peptide-1 receptor agonist (GLP-1 RA) and progressing to addition of rapid-acting meal-time (prandial or bolus) insulin. ${ }^{7,9}$ The effectiveness of insulin therapy and other diabetes medications depends on initiation, appropriate and timely titration, adherence, and persistence. ${ }^{10,11}$

The importance of adherence to insulin therapy is well recognized and has been frequently studied, with nonadherence being a common problem among patients with T2DM. ${ }^{10,12,13}$ However, persistence with insulin therapy, while equally important, is less well studied. ${ }^{14}$ For patients with T2DM prescribed basal insulin, the results of real-world studies suggest that persistence with insulin therapy improves outcomes and lowers health care resource use (HCRU), ${ }^{15}$ while interruption of insulin therapy for $>60$ days increases health care costs. ${ }^{16}$

Patients who ultimately require both basal and bolus insulin therapy face the challenge of maintaining persistence with a regimen requiring multiple daily insulin injections (MDI) ${ }^{7}$ However, information about persistence with MDI and its effect on glycemic control, medical costs, and HCRU in T2DM is limited..$^{14}$ Previous studies have evaluated persistence with initiation of basal insulin or addition of bolus insulin, but not persistence with both insulin types. ${ }^{15-18}$ Moreover, the methods used to define persistence with insulin therapy are variable, making comparisons among studies difficult. ${ }^{2,19}$

Our objectives were to measure persistence with both basal and bolus insulins, using 2 different methods, in patients with T2DM. In addition, we examined the relationship between persistence with MDI and clinical and economic outcomes in a U.S. real-world setting for patients with T2DM.

\section{Methods}

\section{Study Design and Data Source}

We carried out this matched cohort study using the IBM Watson MarketScan Commercial and Medicare Supplemental Claims and Encounters database and the MarketScan Laboratory database (now both included among the IBM MarketScan
Research Databases), ${ }^{20}$ which together contain linked inpatient and outpatient visit data, prescription drug claims data, and enrollment records for a geographically diverse population with commercial and/or retiree health care coverage from more than 100 employers and health plans. The diagnosis and procedure codes used for this study are summarized in Appendix A (available in online article).

The 4-year study period ran from January 1, 2012, through December 31, 2015. During this time, the MarketScan database included approximately 92 million individuals from all 50 U.S. states, and the MarketScan Laboratory database included laboratory testing results from more than 5.3 million individuals linked to the database. ${ }^{20}$ The individuals included in these databases are considered to be representative of the U.S. insured population.

The deidentified data in the MarketScan databases are compliant with the standards of the U.S. Health Insurance Portability and Accountability Act of 1996.

\section{Patients}

Patients eligible for this study had T2DM and were on both basal and bolus insulins (MDI), defining MDI by at least 2 basal and 2 bolus pharmacy claims during a period of 12 consecutive months. The earliest bolus insulin claim that was followed by at least 2 basal and 1 additional bolus insulin claims within the 12-month period was selected as the index claim. Patients were required to be aged 18-64 years on the date of the index claim (index date) and to have at least 6 months of continuous enrollment with medical and pharmacy benefits before the index date (baseline period) and at least 12 months of enrollment after the index date (postindex period). The diagnosis of T2DM was defined as at least 1 inpatient or at least 2 outpatient claims that were at least 30 days and no more than 365 days apart with associated T2DM diagnosis codes in first or second position in the claim (International Classification of Disease, Ninth Revision, Clinical Modification [ICD-9-CM] diagnosis codes $250 . x 0$ or 250.x2, or International Classification of Disease, Tenth Revision, Clinical Modification [ICD-10-CM] diagnosis code E11).

Patients were excluded if they had (a) 1 or more inpatient or outpatient claims with a type 1 diabetes diagnosis (ICD9-CM codes 250.xl and 250.x3 and ICD-10-CM code E10); (b) evidence of pregestational diabetes, gestational diabetes, or pregnancy/labor- and delivery-related diabetes; (c) a diagnosis of cancer/malignant tumors; or (d) any claims for premixed insulin or a GLP-1 RA during the study period. We excluded GLP-1 RA because these agents were infrequently prescribed during the first years of the study period and because we aimed to restrict our analyses to only 1 type of injectable agent (i.e., insulin). Moreover, the full benefit of GLP-1 RAs in the treatment paradigm during the study timeframe was not well established (specifically, cardiovascular disease risk assessment). 


\section{Outcomes and Costs}

Outcomes assessed during the 12-month post-index period included persistence with basal and bolus insulin, annual direct medical costs, annual HCRU, and glycemic control.

We used 2 different methods to evaluate persistence with MDI during the post-index period (Appendix B, available in online article). ${ }^{17}$ For method 1 , we defined persistence as the continuous use of both basal and bolus insulins without a single gap in prescription claims of $>90$ days post-index. Thus, by method 1 , persistence with bolus insulin entailed continuous claims for bolus insulin with no gap of $>90$ days from the index bolus claim or between subsequent claims, and persistence with basal insulin entailed no gap of $>90$ days from the first basal insulin claim within 90 days after the index date and no gap of $>90$ days between subsequent claims over 12 months. For method 2, we defined persistence as the presence of 1 or more claims for both basal and bolus insulin every quarter (every 90 days) for 4 consecutive quarters after the index date (i.e., during the 12-month post-index period).

Annual direct medical costs, annual HCRU, and glycemic control during the post-index year were calculated using method 2 to define persistence.

Annual direct medical costs for inpatient, outpatient, and emergency department (ED) visits were averaged for all causes and for diabetes-related events (i.e., with T2DM diagnosis in any position in the claim). Outpatient costs included ED costs. Annual all-cause and diabetes-related HCRU was assessed, including the proportion of patients with 1 or more hospitalizations and 1 or more ED visits. We did not include outpatient drug expenditures in the cost calculations because of the inherently greater costs associated with persistence with insulin therapy. Therefore, all outpatient drug expenditures were excluded from all-cause medical costs, and diabetes-related drugs were excluded from the diabetes-related medical costs.

Glycemic control was assessed using $\mathrm{HbAlc}$ (Alc) values for the subset of patients who had 1 or more recorded Alc values during both the 6-month baseline period as well as during the last 3 months of the 12-month post-index period. We selected the last 3 months of the post-index period for the follow-up Alc value to allow sufficient time to assess any differences in glycemic control based on persistence with therapy. ${ }^{21}$ We calculated the change from baseline in Alc and the proportion of patients who experienced treatment success, using 2 definitions, namely, (a) a post-index value of Alc $<7 \%$ for those who had baseline $A l c \geq 7 \%$ or (b) a reduction of $\geq 1 \%$ from baseline to follow-up Alc. Multiple Alc values during baseline or follow-up were averaged for individual patients.

\section{Statistical Analysis}

Baseline demographic and clinical characteristics of the unmatched persistent and nonpersistent cohorts, defined using both method 1 and method 2, were summarized using descriptive analyses.

\section{FIGURE 1 Selection of Eligible Patients from} Claims Database

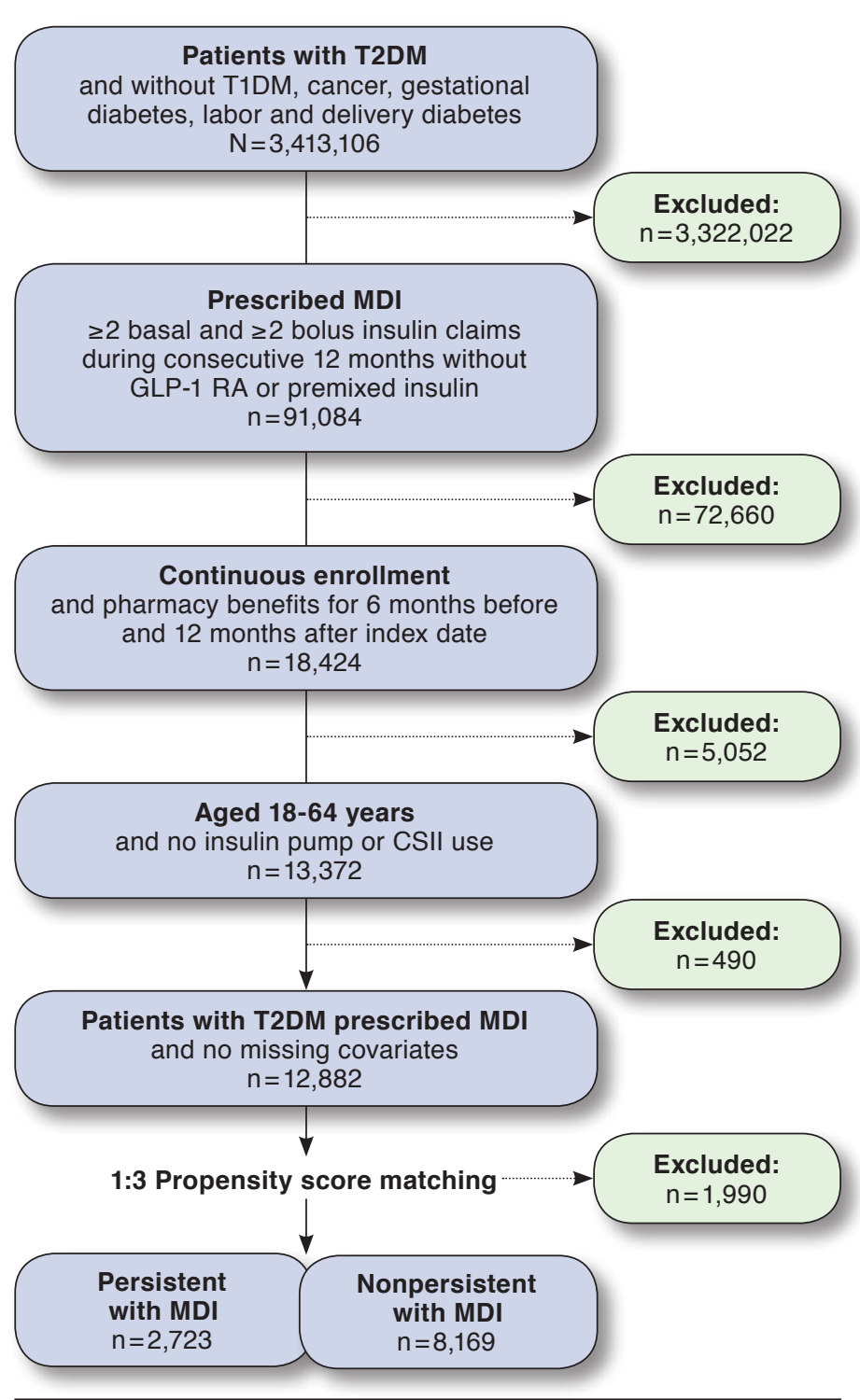

CSII = continuous subcutaneous insulin infusion; GLP-1 RA=glucagon-like peptide-1 receptor agonist; $M D I=$ multiple daily insulin injections; T1DM=type 1 diabetes mellitus; T2DM=type 2 diabetes mellitus.

Using method 2 to define persistence during the post-index year, we conducted propensity score matching to identify matched cohorts of patients persistent and nonpersistent with MDI. The propensity score was defined as the probability of being persistent with MDI therapy during the post-index year and was estimated using logistic regression for patient demographics (age, sex, geographic region, and insurance plan type); the Charlson Comorbidity Index (CCI) score; and 
TABLE 1 Demographic and Clinical Characteristics at Baseline of the Matched Cohorts, by Persistence with MDI During 1 Post-Index Year as Determined Using Method $2^{\mathrm{a}}$

\begin{tabular}{|c|c|c|c|c|c|c|}
\hline \multirow[b]{2}{*}{ Male, n (\%) } & \multicolumn{2}{|c|}{$\begin{array}{l}\text { Persistent with MDI } \\
\quad(\mathrm{n}=2,723)\end{array}$} & \multicolumn{2}{|c|}{$\begin{array}{l}\text { Nonpersistent with MDI } \\
\qquad(\mathrm{n}=8,169)\end{array}$} & \multirow{2}{*}{$\frac{\text { SMD }^{\mathbf{b}}}{0.0198}$} & \multirow{2}{*}{$\frac{P \text { Value }^{\mathrm{c}}}{0.37}$} \\
\hline & 1,577 & $(57.9)$ & 4,651 & $(56.9)$ & & \\
\hline Age at index date, mean (SD) & 52.6 & $(7.8)$ & 52.5 & $(7.9)$ & 0.0028 & 0.90 \\
\hline $18-34$ years, $\mathrm{n}(\%)$ & 69 & $(2.5)$ & 258 & $(3.2)$ & & 0.29 \\
\hline $35-44$ years, $n(\%)$ & 372 & $(13.7)$ & 1,054 & $(12.9)$ & & \\
\hline $45-54$ years, $\mathrm{n}(\%)$ & 973 & $(35.7)$ & 2,890 & $(35.4)$ & & \\
\hline $55-64$ years, $\mathrm{n}(\%)$ & 1,309 & $(48.1)$ & 3,967 & $(48.6)$ & & \\
\hline Geographic region, $\mathrm{n}(\%)$ & & & & & & 0.97 \\
\hline Northeast & 324 & $(11.9)$ & 956 & $(11.7)$ & 0.0059 & \\
\hline North Central & 693 & $(25.4)$ & 2,109 & $(25.8)$ & 0.0084 & \\
\hline South & 1,090 & $(40.0)$ & 3,281 & $(40.2)$ & 0.0027 & \\
\hline West & 616 & $(22.6)$ & 1,823 & $(22.3)$ & 0.0075 & \\
\hline Insurance plan type, n (\%) & & & & & & 0.83 \\
\hline $\mathrm{HMO}$ & 538 & $(19.8)$ & 1,575 & $(19.3)$ & 0.0123 & \\
\hline $\mathrm{PPO}$ & 1,540 & $(56.6)$ & 4,669 & $(57.2)$ & 0.0122 & \\
\hline Other & 645 & $(23.7)$ & 1,925 & $(23.6)$ & 0.0029 & \\
\hline CCI, median (IQR) & 1 & $(1-3)$ & 1 & $(1-3)$ & 0.0073 & 0.74 \\
\hline $0, \mathrm{n}(\%)$ & 245 & $(9.0)$ & 738 & $(9.0)$ & & 0.99 \\
\hline $1, \mathrm{n}(\%)$ & 1,418 & $(52.1)$ & 4,271 & $(52.3)$ & & \\
\hline $2, \mathrm{n}(\%)$ & 367 & $(13.5)$ & 1,107 & $(13.6)$ & & \\
\hline$\geq 3, \mathrm{n}(\%)$ & 693 & $(25.5)$ & 2,053 & $(25.1)$ & & \\
\hline \multicolumn{7}{|l|}{ Comorbidity/complication, n (\%) } \\
\hline Hypertension & 1,494 & $(54.9)$ & 4,444 & $(54.4)$ & 0.0093 & 0.67 \\
\hline Disorder of lipid metabolism & 1,396 & $(51.3)$ & 4,125 & $(50.5)$ & 0.0155 & 0.49 \\
\hline Ischemic heart disease & 438 & $(16.1)$ & 1,315 & $(16.1)$ & 0.0003 & 0.99 \\
\hline Obesity & 384 & $(14.1)$ & 1,136 & $(13.9)$ & 0.0057 & 0.80 \\
\hline Renal disease & 347 & $(12.7)$ & 1,026 & $(12.6)$ & 0.0055 & 0.80 \\
\hline Neuropathy & 325 & $(11.9)$ & 982 & $(12.0)$ & 0.0026 & 0.91 \\
\hline Retinopathy & 233 & $(8.6)$ & 692 & $(8.5)$ & 0.0030 & 0.89 \\
\hline Stroke & 88 & $(3.2)$ & 256 & $(3.1)$ & 0.0052 & 0.80 \\
\hline Acute myocardial infarction & 71 & $(2.6)$ & 221 & $(2.7)$ & 0.0057 & 0.78 \\
\hline Hypoglycemia & 18 & $(0.7)$ & 53 & $(0.7)$ & 0.0016 & 0.95 \\
\hline
\end{tabular}

aPersistence was defined as the presence of 1 or more claims for both basal insulin and bolus insulin every quarter (90 days) for 4 consecutive quarters of the post-index year.

${ }^{b}$ A standardized mean difference $<0.10$ indicates sufficient balance between matched cohorts.

'The chi-square test was used for categorical variables and the t-test for continuous/count variables to compare persistent versus nonpersistent cohorts.

$C C I=$ Charlson Comorbidity Index; HMO = health maintenance organization; $I Q R=$ interquartile range; $M D I=$ multiple daily insulin injections; $P P O=$ preferred provider organization; $S M D=$ standardized mean difference; $S D=$ standard deviation.

11 comorbidities at the index date (hypertension, disorder of lipid metabolism, ischemic heart disease, obesity, renal disease, neuropathy, retinopathy, stroke, acute myocardial infarction, hypoglycemia, and hyperosmolarity). Patients were matched 1:3 with difference in logit of the propensity scores for pairs of individuals from persistent and nonpersistent cohorts of less than or equal to 0.25 times the pooled estimate of the common standard deviation of the logits of the propensity scores (caliper $=0.25$ ). The balance of the measured covariates (demographic information, CCI, and comorbidities/complications) for matched persistent and nonpersistent cohorts was assessed using the chi-square test for categorical variables and t-tests for continuous variables. In addition we calculated the standardized mean difference (SMD) for categorical and continuous variables. An SMD $<0.1$ is considered to represent an acceptable difference between matched cohorts. ${ }^{22,23}$

Bivariate analyses were conducted for comparisons between the persistent cohort and the nonpersistent cohort, and tests for significance were performed based on the distribution of each variable. We conducted chi-square tests for categorical variables and for medical costs, where the distribution was highly skewed, generalized linear model with negative binomial distribution and log link without any covariates. All costs were adjusted to 2015 dollars using the medical care component of the Consumer Price Index. ${ }^{24}$ 
Persistence with Basal-Bolus Insulin Therapy in Patients with Type 2 Diabetes Mellitus and Effect on Clinical and Economic Outcomes: A Retrospective Claims Database Study

TABLE 2 Mean Annual All-Cause and Diabetes-Related Health Care Costs During the Post-Index Year

\begin{tabular}{|c|c|c|c|}
\hline & $\begin{array}{l}\text { Persistent with MDI } \\
(\mathrm{n}=2,723)\end{array}$ & $\begin{array}{l}\text { Nonpersistent with MDI } \\
(\mathrm{n}=8,169)\end{array}$ & $P$ Value \\
\hline \multicolumn{4}{|l|}{ All-cause health care costs } \\
\hline Inpatient, mean (SD)a & $\$ 4,354 \quad(24,333)$ & $\$ 6,946 \quad(38,258)$ & 0.0223 \\
\hline Outpatient, mean (SD) ${ }^{\mathrm{b}}$ & $\$ 9,145 \quad(29,358)$ & $\$ 10,417 \quad(29,620)$ & $<0.0001$ \\
\hline Median (IQR) & $\$ 3,241 \quad(1,276-8,474)$ & $\$ 3,371 \quad(1,284-9,248)$ & \\
\hline $\mathrm{ED}$, mean $(\mathrm{SD})^{\mathrm{a}}$ & $\$ 465 \quad(1,931)$ & $\$ 506 \quad(2,072)$ & 0.51 \\
\hline Total costs, mean (SD) & $\$ 13,499 \quad(41,434)$ & $\$ 17,362 \quad(53,250)$ & $<0.0001$ \\
\hline Median (IQR) & $\$ 3,357 \quad(1,281-10,436)$ & $\$ 3,633 \quad(1,305-12,412)$ & \\
\hline \multicolumn{4}{|l|}{ Diabetes-related health care costs } \\
\hline Inpatient, mean (SD)a & $\$ 2,974 \quad(15,131)$ & $\$ 4,736 \quad(28,780)$ & 0.0434 \\
\hline Outpatient, mean (SD) ${ }^{\mathrm{b}}$ & $\$ 3,417 \quad(8,599)$ & $\$ 3,640 \quad(9,766)$ & 0.0388 \\
\hline Median (IQR) & $\$ 1,103 \quad(564-2,681)$ & $\$ 1,093 \quad(518-2,824)$ & \\
\hline ED, mean $(S D)^{a}$ & $\$ 290 \quad(1,503)$ & $\$ 296 \quad(1,343)$ & 0.91 \\
\hline Total diabetes-related costs, mean (SD) & $\$ 6,392 \quad(18,769)$ & $\$ 8,376 \quad(32,558)$ & $<0.0001$ \\
\hline Median (IQR) & $\$ 1,171 \quad(579-3,329)$ & $\$ 1,170 \quad(537-3,949)$ & \\
\hline Inpatient costs for hospitalized patients & $\mathrm{n}=299 \quad(11.0 \%)$ & $\mathrm{n}=1,196 \quad(14.6 \%)$ & $<0.0001$ \\
\hline Mean (SD) & $\$ 39,651 \quad(63,277)$ & $\$ 47,440 \quad(89,898)$ & 0.0081 \\
\hline Median (IQR) & $\$ 21,466 \quad(11,350-44,608)$ & $\$ 23,562 \quad(12,147-49,107)$ & \\
\hline Diabetes-related inpatient costs & $\mathrm{n}=247 \quad(9.1 \%)$ & $\mathrm{n}=946 \quad(11.6 \%)$ & 0.0003 \\
\hline Mean (SD) & $\$ 32,790 \quad(39,391)$ & $\$ 40,897 \quad(75,358)$ & 0.0020 \\
\hline Median (IQR) & $\$ 19,754 \quad(10,471-39,462)$ & $\$ 21,677 \quad(11,427-41,598)$ & \\
\hline \multicolumn{4}{|c|}{$\begin{array}{l}\text { Note: Costs were in } 2015 \text { U.S. dollars. } \\
\text { a Median inpatient and ED costs were } \$ 0 . \\
\text { boutpatient costs included ED costs. } \\
\text { ED=emergency department; IQR = interquartile range; } M D I=\text { multiple daily insulin injections; SD= standard deviation. }\end{array}$} \\
\hline
\end{tabular}

To compare glycemic control in persistent and nonpersistent patient cohorts, we included all patients from unmatched cohorts with Alc recorded during the baseline period and the last 3 months of the post-index period. (We elected to include all unmatched patients because matching may have further reduced the small size of the patient subset.) The change in Alc from baseline was calculated as the follow-up value minus the baseline value, with the Wilcoxon rank sum test used to evaluate betweencohort differences. Within-group differences (i.e., from baseline to follow-up) were evaluated using the Wilcoxon signed rank test.

Data analyses were conducted using the SAS software package version 9.4 (SAS Institute, Cary, NC). A 2-sided $P$ value of $<0.05$ was considered statistically significant.

\section{Results}

\section{Patients and Persistence with MDI}

We identified 12,882 eligible patients (Figure 1). Approximately one fifth of patients were judged as being persistent with MDI therapy during the post-index year according to each method of assessment, including 2,886 (22.4\%) by method 1 and 2,724 (21.1\%) by method 2. A total of 2,026 patients (15.7\%) were judged persistent by both methods, and 9,298 (72.2\%) were judged nonpersistent by both methods. Baseline characteristics of the unmatched persistent and nonpersistent cohorts were similar as assessed by both methods (data not shown).
After matching the method 2 persistent and nonpersistent cohorts, there were 10,892 patients (85\%) included in the analyses. The matched cohorts were well balanced with regard to demographics and comorbidities at baseline (Table 1).

\section{Annual All-Cause and Diabetes-Related Health Care Costs and Health Care Resource Use}

The mean per-patient all-cause total (inpatient+outpatient, excluding outpatient drug costs) health care costs for patients who were persistent with MDI were significantly lower than for patients who were nonpersistent $(P<0.0001$ for the comparisons; Table 2). Likewise, the all-cause inpatient and outpatient costs, but not the ED costs, were significantly lower for the persistent cohort than for the nonpersistent cohort $(P<0.05)$ A similar pattern was seen for diabetes-related costs (Table 2).

One or more inpatient hospitalizations or ED visits, either all-cause or diabetes-related, were recorded for a lower percentage of patients in the persistent cohort than the nonpersistent cohort, with statistically significant differences between cohorts for all comparisons ( $P \leq 0.005$; Figure 2$)$. Most patients in each cohort had a recorded outpatient visit (99.0\% vs. $99.2 \% ; P=0.40)$ and a recorded diabetes-related outpatient visit (98.2\% vs. $97.2 \% ; P=0.002$ ).

For the 299 and 1,196 patients in persistent and nonpersistent cohorts, respectively, who were hospitalized during the 


\section{FIGURE 2 Annual HCRU According to Persistence with MDI During 1 Post-Index Year: All-Cause and} Diabetes-Related HCRU

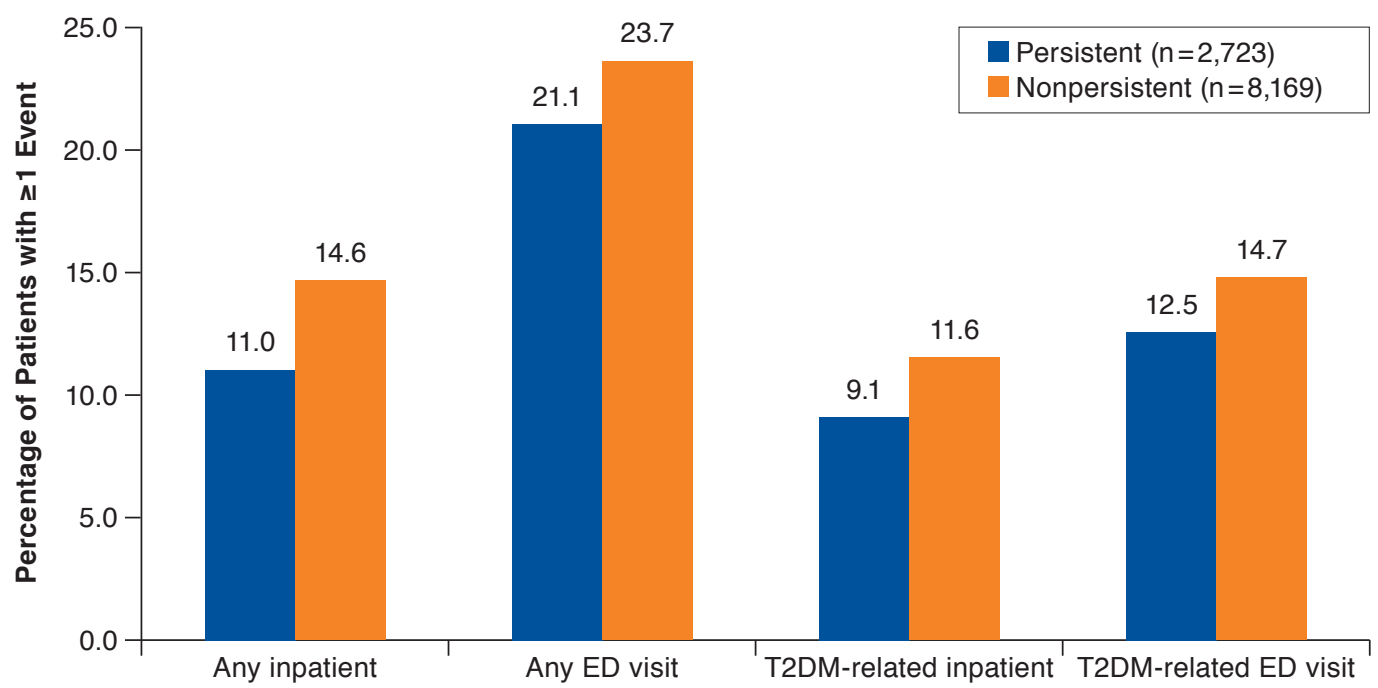

$P \leq 0.005$ for all comparisons between cohorts.

ED = emergency department; HCRU = health care resource use; MDI= multiple daily insulin injections; T2DM=type 2 diabetes mellitus

post-index year, the mean inpatient costs were significantly lower for those in the persistent cohort (Table 2; $P=0.0081$ ). For the subset of 247 and 946 persistent and nonpersistent patients, respectively with a T2DM-related hospitalization, respectively, the mean inpatient costs were also significantly lower in the persistent cohort (Table 2; $P=0.002$ )

\section{Clinical Outcomes}

Recorded Alc values were available during both the 6-month baseline period and the last 3 months of the post-index period for 83 patients (3.0\%) and 248 patients (2.4\%) in unmatched persistent and nonpersistent cohorts, respectively. There was no difference between the 2 cohorts in mean baseline Alc values ( $9.7 \%$ vs. $9.6 \%$, respectively; $P=0.63$; Table 3 ). During the 3 months of the post-index period, mean Alc values were $8.4 \%$ versus $8.8 \%$ in persistent and nonpersistent cohorts, respectively $(P=0.054)$.

A greater proportion of patients who were persistent with MDI (55\%) compared with those nonpersistent with MDI (39\%) achieved treatment success according to 1 or both definitions (Alc decrease from baseline of $\geq 1 \%$ and/or baseline Alc of $\geq 7 \%$ with post-index Alc $<7 \%$; Table 3). Moreover, patients who were persistent with MDI experienced a greater mean reduction in Alc from baseline (-1.3\% vs. $-0.8 \% ; P=0.006)$.

\section{Discussion}

We found that poor persistence with MDI was prevalent as defined by both methods among patients included in this matched cohort, claims database study. Only one fifth of patients were persistent with both basal and bolus insulin therapy during the 12-month post-index period, and outcomes were statistically significantly better for these patients than for those with poor persistence. Moreover, persistence with MDI was associated with lower mean annual medical costs (excluding outpatient drug costs), and lower HCRU during the post-index period. In the small subpopulation with available Alc test results, persistence with MDI was associated with a greater mean reduction in Alc and greater likelihood of achieving treatment success.

The low persistence with MDI that we identified using either method is in contrast with results of 2 earlier studies evaluating persistence with a single insulin type (prandial/bolus insulin) that used the same 2 methods we used but reported divergent results for these 2 persistence measures., ${ }^{17,18}$ Bonafede et al. (2011) found that measured persistence with newly instituted rapid-acting insulin added to a basal regimen was greater using method 2 at 1 year (42\%, vs. 19\% using method 1$){ }^{17}$ Similarly, Sambamoorthi et al. (2016) found that method 2 defined greater persistence with newly instituted rapid-acting insulin added to an OAD regimen (at 1 year, 34\% vs. 18\% using method 1). ${ }^{18}$ Both groups of authors speculated that method 1 might be less reliable for defining persistence while physicians are titrating the rapid-acting insulin dose because, for example, a >90-day gap might not mean discontinuation of therapy. Although their findings could be construed as favoring use of method 2 (presence of 1 or more claims every quarter for 


\begin{tabular}{|c|c|c|c|}
\hline \multirow[t]{2}{*}{ TABLE 3} & \multicolumn{3}{|c|}{$\begin{array}{l}\text { A1c Results for Patients with Recorded } \\
\text { Alc During 6-Month Baseline Period } \\
\text { and Last } 3 \text { Months of Post-Index Year }\end{array}$} \\
\hline & $\begin{array}{c}\text { Persistent with } \\
\text { MDI } \\
(\mathrm{n}=83)\end{array}$ & $\begin{array}{l}\text { Nonpersistent } \\
\text { with MDI } \\
(\mathrm{n}=248)\end{array}$ & $P$ Value ${ }^{a}$ \\
\hline \multicolumn{4}{|l|}{ Baseline Alc } \\
\hline Alc $(\%)$, mean $(\mathrm{SD})$ & $9.67 \quad(1.9)$ & $9.60(2.1)$ & 0.631 \\
\hline $\begin{array}{l}\text { Alc (\%), median } \\
\text { (range) }\end{array}$ & $9.2(6.5-17.2)$ & $9.2(5.9-18.2)$ & \\
\hline \multicolumn{4}{|l|}{ Post-index Alc } \\
\hline Alc $(\%)$, mean $(\mathrm{SD})$ & $8.38(1.5)$ & $8.84(1.8)$ & 0.054 \\
\hline $\begin{array}{l}\text { Alc (\%), median } \\
\text { (range) }\end{array}$ & $8.0(5.6-12.6)$ & $8.4(5.2-14.9)$ & \\
\hline \multicolumn{4}{|l|}{ Change from baseline } \\
\hline $\begin{array}{l}\text { Mean (SD) change in } \\
\text { Alc, \% }\end{array}$ & $-1.29(2.0)^{b}$ & $-0.76(2.1)^{b}$ & 0.006 \\
\hline $\begin{array}{l}\text { Median (range) } \\
\text { change in Alc, \% }\end{array}$ & $-1.0(-8.9$ to 5.6$)$ & $-0.45(-9.4$ to 5.8$)$ & \\
\hline \multicolumn{4}{|l|}{ Treatment success, n (\%) } \\
\hline $\begin{array}{l}\text { Alc decrease } \geq 1 \% \\
\text { from baseline }\end{array}$ & $44(53.0)$ & $94(37.9)$ & \\
\hline $\begin{array}{l}\text { Alc baseline }>7 \% \& \\
\text { post-index } \leq 7 \%\end{array}$ & $11(13.3)$ & $19(7.7)$ & \\
\hline $\begin{array}{l}\text { Either definition of } \\
\text { treatment success }\end{array}$ & $46(55.4)$ & $97(39.1)$ & 0.009 \\
\hline \multicolumn{4}{|c|}{$\begin{array}{l}\text { aThe chi-square test was used to compare categorical variables, and the Wilcoxon } \\
\text { rank sum was used to compare continuous variables. } \\
b P<0.0001 \text { (Wilcoxon signed rank test) for within-cohort change from mean base- } \\
\text { line Alc to mean Alc value during the past } 3 \text { months of the post-index year. } \\
\text { Alc=hemoglobin Alc; MDI= multiple daily insulin injections; } S D=\text { standard deviation. }\end{array}$} \\
\hline
\end{tabular}

4 consecutive quarters), we elected to use both methods to define persistence because of the lack of data regarding persistence with both basal and bolus insulins together.

Earlier studies examining persistence with a single insulin type have reported widely variable results ranging from $29 \%$ to $69 \%$ persistence with basal insulin at 1 year after initiation $^{16,25-27}$ and from $18 \%$ to $34 \%$ persistence with rapid-acting or prandial insulin at 1 year after initiation. ${ }^{17,18,28}$ Possible reasons for the wide variation include different study populations (e.g., elderly vs. all adults) and the different measures of persistence used to address the challenge of determining persistence with an injectable agent such as insulin via prescription or claims records., ${ }^{2,1419}$ As described in 2 systematic reviews, reported measures of persistence have included mean times to nonpersistence, defined using varying times without a gap between prescription refills (usually 60 or 90 days), a prespecified number of refills within a prespecified time period, the percentages of patients without a treatment gap, and the hazard or odds of nonpersistence. ${ }^{2,19}$ This variability in persistence definitions has resulted in calls for standardizing methods and identifying a universal persistence measure..$^{1,2,19,29}$ Further studies are needed to explore other lengths of gaps in claims as measures of persistence with insulin.

The importance of defining persistence is illustrated by results of a recent study finding that nonoptimization of medications results in annual costs of drug-related morbidity and mortality of $\$ 528.4$ billion (in 2016 dollars). ${ }^{30}$ In retrospective analyses of medication adherence and persistence for T2DM, the higher medication costs of adherence were sufficiently offset by savings in other health care areas to result in lower total health care costs, whereas persistence was associated with more variable effects on total health care costs, with some studies finding no reduction in total costs. ${ }^{31}$ In this analysis, because the study was not designed to capture long-term health care costs, we excluded outpatient drug costs from the cost analyses because persistence with MDI and, thus, the short-term total health care costs would, by definition, increase in the persistent cohort but would not reflect potential cost offsets of long-term medication persistence.

We speculate that although the initial medical costs of persistence including drug costs may be greater than costs associated with poor persistence, the benefits of maintaining glycemic control should result in lower long-term medical costs over time. We acknowledge the limitations of analyzing retrospective data; however, extrapolation of the data presented here would imply lower costs year over year, provided there are no substantial changes in claims.

Moreover, although our study did not test the association of glycemic control and overall medical costs, Stephens et al. (2006) in their review found that improved glycemic control was associated with lower overall medical costs for patients with diabetes in managed care plans. ${ }^{32}$ Results of more recent studies have supported these conclusions in broader populations, with findings that HCRU and total medical costs are lower in association with better adherence to and persistence with antidiabetic therapy and despite higher pharmacy and outpatient costs in 1 study. 33,34

Similarly, the results of economic modeling suggest that cost savings can be achieved with earlier progression to insulin in T2DM by avoiding the costs associated with diabetesrelated complications and mortality. ${ }^{35}$ However, in another review, health resource use was lower, but costs not consistently so, with improved adherence to antidiabetic therapy. ${ }^{36}$ Nonetheless, these findings may not be applicable to populations such as the one we studied nor to other populations with advanced/severe T2DM.

The high prevalence (almost 80\%) of poor persistence with both basal and bolus insulin therapy highlights the need for interventions to improve persistence with pharmacotherapy in T2DM. Although it is outside the scope of this article to provide a detailed discussion of possible interventions to improve persistence, such interventions could include measures to address the factors associated with insulin omission, such as 
injection burden, use of multiple insulins and supplies, emotional and behavioral issues, and the interference with daily activities..$^{10,12-14}$ Interventions such as durable insulin pumps and tube-free insulin delivery devices are other options for people with T2DM who require intensive insulin therapy. ${ }^{37-41}$ In addition, optimizing physician-patient communication and interventions such as patient support programs can improve medication persistence. ${ }^{14,42}$

This study was unique in measuring persistence with 2 types of insulin prescribed concomitantly for patients with T2DM, adding to the limited published information available regarding patients prescribed MDI. The matched cohort analyses enabled us to compare outcomes for patients matched on demographic and clinical characteristics at baseline. Other strengths of the study include the large patient population, use of a well-maintained claims database, the requirement for 18 months of continuous enrollment with follow-up for 1 full year, and the use of 2 different methods to define persistence.

\section{Limitations}

This study has some limitations to consider. The IBM MarketScan databases include claims data for insured individuals and their families; therefore, these results cannot be generalized to populations other than those with similar commercial health care coverage. Moreover, the prescribing information is limited, with no details on insulin dosage, and we cannot be certain that patients actually took medications that were prescribed. For example, depending on their insurance co-pay, patients may stockpile their insulin or take partial doses. Therefore our data may underestimate the problem of poor persistence. Conversely, we did not analyze switches from insulin to other antidiabetic medications, for example, discontinuation of a prandial insulin in favor of GLP-1 RA or an oral agent, which may overestimate poor persistence with antidiabetic therapy. In addition, we did not examine whether discontinuations were associated with health care plan changes in drug coverage.

The 18-month study timeline, while appropriate to examine persistence over 1 year of MDI therapy, was insufficient to assess the development of diabetes-related complications over time. Moreover, only a minority of patients had baseline and post-index Alc values, which limited our ability to extrapolate findings for glycemic control in relation to basal and bolus insulin persistence. In addition, it would have been ideal to follow patients after the post-index year to assess Alc; however, requiring further continuous enrollment and Alc would have further reduced the sample size. Moreover, adding follow-up time after the 12-month index period would have required assessment of other outcomes of interest. For these reasons, we made a decision to select a time frame within the study period (last 3 months to ensure consistency among the cohorts).
Other study limitations included claims data that may not have reflected actual clinical practice and did not capture details of physician-patient communications or patient-related factors such as injection technique. Moreover, claims data contain no details about health-related behaviors that could influence T2DM-related outcomes, such as diet and exercise. Finally, the cost analyses were limited to direct medical costs, with no information available about indirect costs, such as those associated with absenteeism and presenteeism.

\section{Conclusions}

This study found that only $21 \%-22 \%$ of patients with T2DM were persistent with both basal and bolus insulin therapy over 1 year as defined by 2 different methods. Poor persistence with MDI was associated with greater medical costs, greater HCRU, and poorer glycemic control as compared with persistence among matched patient cohorts. Further research is necessary to standardize the definition of persistence using electronic databases, as well as to identify factors associated with insulin nonpersistence. Interventions are needed to improve basal and bolus insulin persistence in patients with T2DM, by reducing injection burden and treatment-related supplies, including use of multiple insulins, to aid patients with T2DM to maintain glycemic control and subsequently reduce HCRU and health care expenditures.

\section{Authors}

STEVEN V. EDELMAN, MD, Division of Endocrinology and Metabolism, University of California School of Medicine, San Diego; Veterans Affairs Medical Center, San Diego, California; and Taking Control of Your Diabetes, Del Mar, California. ANASTASIA ERMAKOVA, PharmD; YAN XIONG, MS; RAY SIERADZAN, PharmD; and STEPHANIE D. TAYLOR, PhD, Becton, Dickinson and Co., Franklin Lakes, New Jersey.

AUTHOR CORRESPONDENCE: Stephanie D. Taylor, PhD, Health Economics and Outcomes Research, Becton, Dickinson and Co., 1 Becton Dr., Franklin Lakes, NJ 07417. Tel.: 201.847.5714;

E-mail: Stephanie.Taylor@bd.com.

\section{DISCLOSURES}

Funding for this study was provided by Becton, Dickinson and Company (BD). All authors except Edelman are employees and stockholders of BD. Edelman reports board membership at Senseonics and participation in advisory board/speakers bureau at Lilly USA, MannKind, Novo Nordisk, SanofiAventis U.S., Merck, and AstraZeneca, all unrelated to this study.

A poster for this study was presented at the AMCP Managed Care \& Specialty Pharmacy Annual Meeting 2018; April 23-26, 2018; Boston MA.

\section{ACKNOWLEDGMENTS}

Medical writing and editorial assistance was provided by Elizabeth V. Hillyer, DVM, and funded by BD 


\section{Persistence with Basal-Bolus Insulin Therapy in Patients with Type 2 Diabetes Mellitus and Effect on Clinical and Economic Outcomes: A Retrospective Claims Database Study}

\section{REFERENCES}

1. World Health Organization (WHO). Adherence to long-term therapies: evidence for action. 2003. Available at: http://www.who.int/chp/knowledge/ publications/adherence_report/en/. Accessed August 14, 2019

2. McGovern A, Tippu Z, Hinton W, Munro N, Whyte M, de Lusignan S. Comparison of medication adherence and persistence in type 2 diabetes: a systematic review and meta-analysis. Diabetes Obes Metab. 2018;20(4):1040-43.

3. Cramer JA, Roy A, Burrell A, et al. Medication compliance and persistence: terminology and definitions. Value Health. 2008;11(1):44-47.

4. American Diabetes Association. Economic costs of diabetes in the U.S. in 2017. Diabetes Care. 2018;41(5):917-28.

5. Bommer C, Heesemann E, Sagalova V, et al. The global economic burden of diabetes in adults aged 20-79 years: a cost-of-illness study. Lancet Diabetes Endocrinol. 2017;5(6):423-30.

6. Centers for Disease Control and Prevention (CDC). National Diabetes Statistics Report, 2017: estimates of diabetes and its burden in the United States. Last reviewed February 24, 2018. Available at: https://www.cdc.gov/ diabetes/data/statistics/statistics-report.html. Accessed August 14, 2019.

7. Davies MJ, D'Alessio DA, Fradkin J, et al. Management of hyperglycemia in type 2 diabetes, 2018: a consensus report by the American Diabetes Association (ADA) and the European Association for the Study of Diabetes (EASD). Diabetes Care. 2018;41(12):2669-01.

8. Li R, Bilik D, Brown MB, et al. Medical costs associated with type 2 diabetes complications and comorbidities. Am J Manag Care. 2013;19(5):421-30.

9. American Diabetes Association. 8. Pharmacologic approaches to glycemic treatment: Standards of Medical Care in Diabetes-2018. Diabetes Care. 2018;41(Suppl 1):S73-S85

10. Peyrot M, Barnett AH, Meneghini LF, Schumm-Draeger PM. Insulin adherence behaviours and barriers in the multinational Global Attitudes of Patients and Physicians in Insulin Therapy study. Diabet Med. 2012;29(5):682-89.

11. Peyrot M, Rubin RR. Perceived medication benefits and their association with interest in using inhaled insulin in type 2 diabetes: a model of patients cognitive framework. Patient Prefer Adherence. 2011;5:255-65.

12. Peyrot M, Rubin RR, Kruger DF, Travis LB. Correlates of insulin injection omission. Diabetes Care. 2010;33(2):240-45.

13. Peyrot M, Barnett AH, Meneghini LF, Schumm-Draeger PM. Factors associated with injection omission/non-adherence in the Global Attitudes of Patients and Physicians in Insulin Therapy study. Diabetes Obes Metab. 2012;14(12):1081-87.

14. Garnero TL, Davis NJ, Perez-Nieves M, et al. Insulin non-persistence among people with type 2 diabetes: how to get your patients to stay on insulin therapy. Postgrad Med. 2018;130(4):394-401.

15. Wei W, Pan C, Xie L, Baser O. Real-world insulin treatment persistence among patients with type 2 diabetes. Endocr Pract. 2014;20(1):52-61

16. Kalirai S, Duan R, Liu D, Reed BL. Economic impact of treatment duration and persistence with basal insulin in previously insulin-naive users. J Manag Care Spec Pharm. 2017;23(3):327-36. Available at: https://www.jmcp. org/doi/10.18553/jmcp.2017.23.3.327.

17. Bonafede MM, Kalsekar A, Pawaskar M, et al. Insulin use and persistence in patients with type 2 diabetes adding mealtime insulin to a basal regimen: a retrospective database analysis. BMC Endocr Disord. 2011;11:3.

18. Sambamoorthi U, Deb A, Zhou S, Garg R, Fan T, Boss A. Rapid acting insulin use and persistence among elderly type 2 diabetes patients adding RAI to oral antidiabetes drug regimens. J Diabetes Res. 2016;2016:5374931

19. Stolpe S, Kroes MA, Webb N, Wisniewski T. A systematic review of insulin adherence measures in patients with diabetes. J Manag Care Spec Pharm. 2016;22(11):1224-46. Available at: https://www.jmcp.org/doi/10.18553/ jmcp.2016.22.11.1224.
20. IBM Watson Health. IBM MarketScan Research Databases. March 18 2019. Available at: https://www.ibm.com/us-en/marketplace/marketscanresearch-databases. Accessed August 12, 2019.

21. Buysman EK, Liu F, Hammer M, Langer J. Impact of medication adherence and persistence on clinical and economic outcomes in patients with type 2 diabetes treated with liraglutide: a retrospective cohort study. Adv Ther. 2015;32(4):341-55.

22. Austin PC. An introduction to propensity score methods for reducing the effects of confounding in observational studies. Multivariate Behav Res. 2011;46(3):399-424.

23. Linden A, Samuels SJ. Using balance statistics to determine the optimal number of controls in matching studies. J Eval Clin Pract. 2013;19(5):968-75.

24. U.S. Department of Labor, Bureau of Labor Statistics. Consumer Price Index. All urban consumers. August 14, 2019. Available at: https://data.bls. gov/timeseries/CUUR0000SAM?output_view=pct_12mths. Accessed August 14, 2019.

25. Zhou FL, Xie L, Pan C, et al. Relationship between treatment persistence and AlC trends among patients with type 2 diabetes newly initiating basal insulin. Diabetes Obes Metab. 2018;20(5):1298-301.

26. Wei W, Jiang J, Lou Y, Ganguli S, Matusik MS. Benchmarking insulin treatment persistence among patients with type 2 diabetes across different U.S. payer segments. J Manag Care Spec Pharm. 2017;23(3):278-90. Available at: https://www.jmcp.org/doi/10.18553/jmcp.2017.16227.

27. Cooke CE, Lee HY, Tong YP, Haines ST. Persistence with injectable antidiabetic agents in members with type 2 diabetes in a commercial managed care organization. Curr Med Res Opin. 2010;26(1):231-38.

28. Sambamoorthi U, Garg R, Deb A, Fan T, Boss A. Persistence with rapid-acting insulin and its association with $\mathrm{AlC}$ level and severe hypoglycemia among elderly patients with type 2 diabetes. Curr Med Res Opin 2017;33(7):1309-16.

29. Raebel MA, Schmittdiel J, Karter AJ, Konieczny JL, Steiner JF. Standardizing terminology and definitions of medication adherence and persistence in research employing electronic databases. Med Care. 2013;51 (8 Suppl 3):S11-21.

30. Watanabe JH, McInnis T, Hirsch JD. Cost of prescription drug-related morbidity and mortality. Ann Pharmacother. 2018;52(9):829-37.

31. Kennedy-Martin T, Boye KS, Peng X. Cost of medication adherence and persistence in type 2 diabetes mellitus: a literature review. Patient Prefer Adherence. 2017;11:1103-17.

32. Stephens JM, Botteman MF, Hay JW. Economic impact of antidiabetic medications and glycemic control on managed care organizations: a review of the literature. J Manag Care Pharm. 2006;12(2):130-42. Available at: https://www.jmcp.org/doi/10.18553/jmcp.2006.12.2.130.

33. Perez-Nieves M, Kabul S, Desai U, et al. Basal insulin persistence, associated factors, and outcomes after treatment initiation among people with type 2 diabetes mellitus in the U.S. Curr Med Res Opin. 2016;32(4):669-80.

34. Egede LE, Gebregziabher M, Dismuke CE, et al. Medication nonadherence in diabetes: longitudinal effects on costs and potential cost savings from improvement. Diabetes Care. 2012;35(12):2533-39.

35. Smolen HJ, Murphy DR, Gahn JC, Yu X, Curtis BH. The evaluation of clinical and cost outcomes associated with earlier initiation of insulin in patients with type 2 diabetes mellitus. J Manag Care Spec Pharm. 2014;20(9):968-84. Available at: https://www.jmcp.org/doi/10.18553/ jmcp.2014.20.9.968.

36. Asche C, LaFleur J, Conner C. A review of diabetes treatment adherence and the association with clinical and economic outcomes. Clin Ther 2011;33(1):74-109.

37. Aronson R, Reznik Y, Conget I, et al. Sustained efficacy of insulin pump therapy compared with multiple daily injections in type 2 diabetes: 12-month data from the OpT2mise randomized trial. Diabetes Obes Metab. 2016;18(5):500-07. 
Persistence with Basal-Bolus Insulin Therapy in Patients with Type 2 Diabetes Mellitus

and Effect on Clinical and Economic Outcomes: A Retrospective Claims Database Study

38. Winter A, Lintner M, Knezevich E. V-Go insulin delivery system versus multiple daily insulin injections for patients with uncontrolled type 2 diabetes mellitus. J Diabetes Sci Technol. 2015;9(5):1111-16.

39. Polonsky WH, Fisher L, Hessler D, Edelman SV. Development of a new measure for assessing insulin delivery device satisfaction in patients with type 1 and type 2 diabetes. Diabetes Technol Ther. 2015;17(11):773-79.

40. Layne JE, Parkin CG, Zisser H. Efficacy of a tubeless patch pump in patients with type 2 diabetes previously treated with multiple daily injections. J Diabetes Sci Technol. 2017;11(1):178-79.
41. Lajara R, Nikkel C, Abbott S. The clinical and economic impact of the $\mathrm{V}-\mathrm{Go}((\mathrm{R}))$ disposable insulin delivery device for insulin delivery in patients with poorly controlled diabetes at high risk. Drugs Real World Outcomes. 2016;3(2):191-99.

42. Goldman JD, Gill J, Horn T, Reid T, Strong J, Polonsky WH. Improved treatment engagement among patients with diabetes treated with insulin glargine $300 \mathrm{U} / \mathrm{mL}$ who participated in the $\mathrm{COACH}$ support program. Diabetes Ther. 2018;9(5):2143-53. 
Persistence with Basal-Bolus Insulin Therapy in Patients with Type 2 Diabetes Mellitus and Effect on Clinical and Economic Outcomes: A Retrospective Claims Database Study

\section{APPENDIX A Code Lists Used in this Study}

\begin{tabular}{|c|c|c|}
\hline Comorbid Conditions & ICD-9-CM & ICD-10-CM \\
\hline Type 1 diabetes mellitus & $250 . x 1,250 . x 3$ & E10 \\
\hline Type 2 diabetes mellitus & $250 . x 0,250 . x 2$ & Ell \\
\hline Obesity & $278, V 85.3-V 85.45$ & E66 (Overweight and obesity), Z68.3-Z68.45 \\
\hline Disorder of lipid metabolism & 272 & E78 \\
\hline Hypertension & 401 & $\mathrm{I} 10$ \\
\hline \multicolumn{3}{|l|}{ Diabetes-related Complications } \\
\hline Diabetic neuropathy (micro) & $250.6 x, 357.2,356 . x$ & E11.4x, E10.4x, E08.4x, E13.4x, G60.X \\
\hline Renal disease (micro) & $\begin{array}{l}250.4 x, 403 . x x, 404 . x x, 405.01,405.11,405.91,581 . \\
x x, 582 x x 583 x x, 585 . x x, 586 . x x, 588 . x x, 791.1 x \\
\text { V42.0, V45.1, V56x }\end{array}$ & $\begin{array}{l}\text { E11.21, E11.22, E1129, E13.21, E13.22, E1329, } \\
\text { E10.21, E10.22, E1029, I129, I120, I1310, I130, } \\
\text { I1311, I132, I150, N03, N05, N044, N022, N043, } \\
\text { N040, N08, N048, N049, N181, N182, N183, } \\
\text { N184, N185, N186, N189, N19, N250, N251, } \\
\text { N2581, N2589, N259, R803, R809, Z49, Z94.0, } \\
\text { Z99.2 }\end{array}$ \\
\hline Retinopathy (micro) & $250.5 x, 362 . x, 366.41,249.50$ & E113x, E103x, E13.3x, H35 \\
\hline Acute MI, previous MI, (macro) & 410,412 & $\mathrm{I} 21 \mathrm{x}, \mathrm{I} 22 \mathrm{x}, \mathrm{I} 252$ \\
\hline Stroke/CVA (macro) & $430 . \mathrm{xx}-438 . \mathrm{xx}$ & I609x-I69x, G45x, G46x \\
\hline Ischemic heart disease, PAD, PVD, CHF (macro) & $\begin{array}{l}398.91,402.01,402.11,402.91,404.01,404.03 \\
404.11,404.13,404.91,404.93,425.4-425.9,411 \\
413,414,250.7 x, 443.81,428 x, 440 x, 441 x, 44389 \\
4439,4771\end{array}$ & $\begin{array}{l}\mathrm{I} 24, \mathrm{I} 20, \mathrm{I} 25, \mathrm{E} 1151, \mathrm{E} 1051, \mathrm{I} 798, \mathrm{I} 09.9, \mathrm{I} 11.0, \\
\mathrm{I} 13.0, \mathrm{I} 113.2, \mathrm{I} 25.5, \mathrm{I} 42.0, \mathrm{I} 42.5-\mathrm{I} 42.9, \mathrm{I} 43 . x, \mathrm{I} 50 . x \\
\mathrm{I} 70 \mathrm{x}, \mathrm{I} 71 \mathrm{x}, \mathrm{I} 73.89, \mathrm{I} 73.9, \mathrm{I} 771,\end{array}$ \\
\hline Hypoglycemia & $2511,2512,25030$ & E160, E161, E162, E11641, E11649 \\
\hline Hyperosmolarity & $25020,25022,25032,25012$ & E1100, E1101 \\
\hline \multicolumn{3}{|l|}{ Other Conditions } \\
\hline Cancer/malignant tumors & $\begin{array}{l}\text { 140.xx-172.xx, 174.xx-195.xx, 196.xx-198.xx, 199.xx, } \\
\text { 200.xx-208.xx, 209.0x-209.3x, 230.xx-234.xx }\end{array}$ & C00-D49 (exclude D10-D36 and D3A) \\
\hline $\begin{array}{l}\text { Labor and delivery, Pregnancy, gestational, } \\
\text { pregestational, DM }\end{array}$ & $\begin{array}{l}630-679 \text { (includes gestational and pregestational } \\
\text { codes } 648.0 x, 648.8 x \text { ), V220, V221, V222 }\end{array}$ & $\begin{array}{l}\text { O00-O16, 020-048 (includes gestational and } \\
\text { pregestational codes O24.3x, O24.9x, O24.4x), } \\
\text { O60-O77, O80-O82, O85-O92, O94-O9A, Z34, } \\
\mathrm{Z33,Z3A}\end{array}$ \\
\hline
\end{tabular}

CHF= congestive heart failure; CVA = cerebrovascular accident; DM= diabetes mellitus; ICD-9-CM=International Classification of Diseases, Ninth Revision, Clinical Modification; ICD-10-CM=International Classification of Diseases, 10th Revision, Clinical Modification; MI=myocardial infarction; PAD= peripheral artery disease; $P V D=$ peripheral vascular disease. 
Persistence with Basal-Bolus Insulin Therapy in Patients with Type 2 Diabetes Mellitus and Effect on Clinical and Economic Outcomes: A Retrospective Claims Database Study

APPENDIX B Study Design and Depiction of the 2 Methods to Evaluate Persistence with Basal-Bolus Insulin Therapy

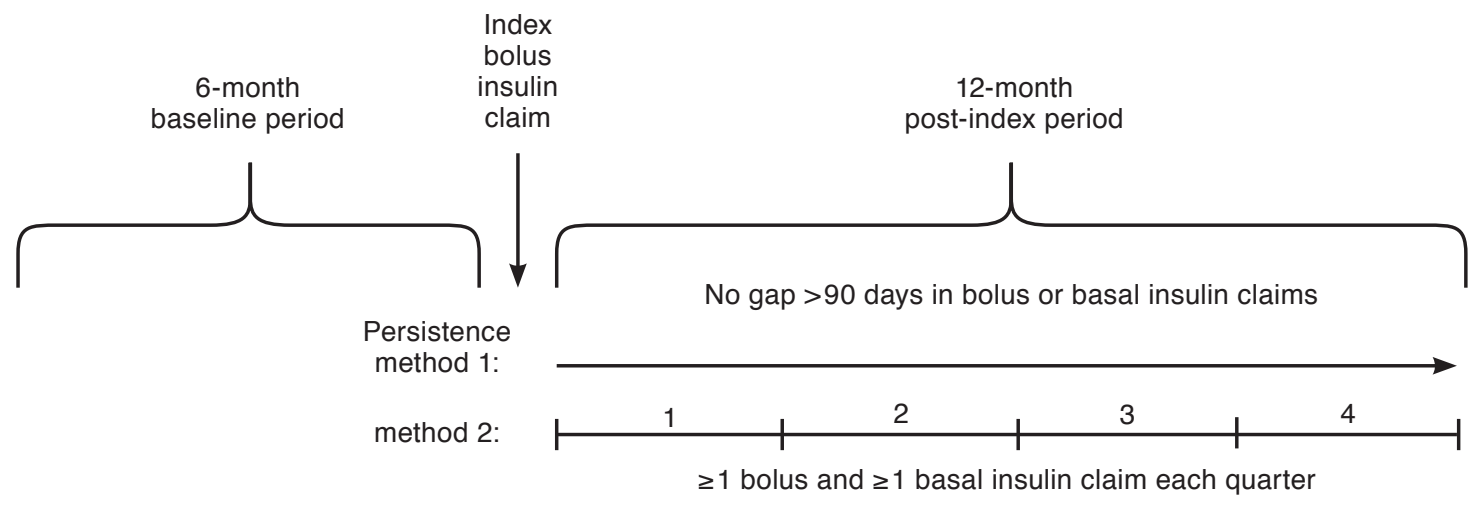

18 months of continuous enrollment 